\title{
Growth and Characterization of Organic NLO Material
}

\author{
Dr. J. Thomas Joseph Prakash \\ Assistant Professor, \\ Department of Physics, \\ H.H. The Rajah's Government Arts College, \\ Pudukkottai, Tamil Nadu, India.
}

\author{
J. Felicita Vimala \\ Lecturer in Physics, \\ DIET, \\ Mayanur, Karur, \\ Tamilnadu, India
}

\author{
M. Lawrence \\ Assistant Professor, \\ Department of Physics, \\ Kurinji College of Arts and Science, \\ Tiruchirappalli, Tamil Nadu, India
}

\begin{abstract}
Single crystals of Meta nitro aniline (mNA), an Organic nonlinear optical (NLO) material, were grown by slow solvent evaporation technique at room temperature. Acetone was used as solvent. The mNA crystals obtained by the above technique were subjected to different characterization analyses. The lattice dimensions were determined from the single crystal x-ray diffraction analysis. The functional groups and optical behavior of the crystal was identified from FTIR and UV-vis analysis. Micro hardness and etching studies were also carried out on the sample respectively.
\end{abstract}

Keywords: X-ray diffraction; Growth from solutions; nonlinear optic materials

\section{INTRODUCTION}

Organic crystals have been shown to have potential applications in nonlinear optics. Nonlinear optics (NLO) is at the forefront of current research because of its importance in providing the key functions of frequency shifting optical modulation, optical switching, optical logic, and optical memory for the emerging techniques in areas such as telecommunications, signal processing, and optical interconnections [4, 12 \& 13]. Organic materials have been of particular interest because the nonlinear optical response in this broad class of materials is microscopic in origin, offering an opportunity to use theoretical modeling coupled with synthetic flexibility to design and produce novel materials [8,10 \& 11]. Organic NLO materials have a very large nonlinear susceptibility, which are in many cases several orders of magnitude higher than that of inorganic crystals such as $\mathrm{LiNbo}_{3}, \mathrm{KNbO}_{3}$ and potassium dihydrogen phosphate (KDP) [5, 18]. Methyl-para-hydroxy benzoate (MHB) is an organic NLO material. In the study, it is reported that the second harmonic generation (SHG) efficiency of MHB crystal was 40 times that of urea [15]. By molecular engineering, develop many organic crystals displaying better nonlinear optical properties than the inorganic materials, in particular for second Harmonic Generation (SHG) [7, 14]. Current interest in optical communication systems has stimulated investigation of new, effective organic crystals for SHG and electro-optic modulation [2, 9]. Most of these molecules show large nonlinear optical responses, with the electron-donor and electron-acceptor groups located at the extreme of a system involving correlated and high delocalized $\pi$ electron states [3]. Single crystal of mNA were grown by vertical Bridgeman method and vapor growth method, but the former has the problem of cleavages along (010) plane of growth, which has constraints of pocked and reticular grown interfaces at fast withdrawal rates [1]. mNA crystals have been grown using benzene-acetone as mixed solvents by solvent evaporation technique [6]. In present paper, we report the conditions for the growth of good quality single crystal of mNA by slow evaporation method using acetone as solvent. The grown single crystal of mNA was subjected to characterization techniques like X-ray diffraction, FT-IR UV-Vis-NIR spectroscopy, etching and micro hardness.

\section{CRYSTAL STRUCTURE OF MNA}

The molecular formula of m-nitro aniline has $\mathrm{C}_{6} \mathrm{H}_{6} \mathrm{~N}_{2} \mathrm{O}_{2}$ and its melting point is $112{ }^{\circ} \mathrm{C}$. The mNA crystallizes in an orthorhombic unit cell of space group $\mathrm{Pbc}_{21}$ and point group $\mathrm{mm} 2$. The mNA crystal is negative biaxial crystal with principal dielectric axes $\mathrm{X}, \mathrm{Y}$ and $\mathrm{Z}$ parallel to crystallographic axes ' $\mathrm{c}$ ', ' $b$ ' and ' $a$ ' respectively. The molecular structure of mNA is shown in Figure 1a. The molecule is essentially planar, with the dipole moment directed from $\mathrm{N} 1$ in the amino group (donor) towards the N2 (acceptor) in the nitro group. The projection of mNA crystal structure along the $\{100\}$ direction is shown in Figure 1c. The molecular structure of mNA crystal is as shown in Figure 1d. mNA has four molecules per unit cell of dimensions $\mathrm{a}=6.501 \mathrm{~A}^{\circ}, \mathrm{b}=19.330 \mathrm{~A}^{\circ}$ and $\mathrm{c}=5.082 \mathrm{~A}^{\circ}$. The crystal packing in the unit cell is represented in the Figure 1b. A close observation of this figure reveals that the hydrogen rich faces containing amino groups are exposed along (001) plane and oxygen rich faces containing nitro groups are exposed along (001) plane.

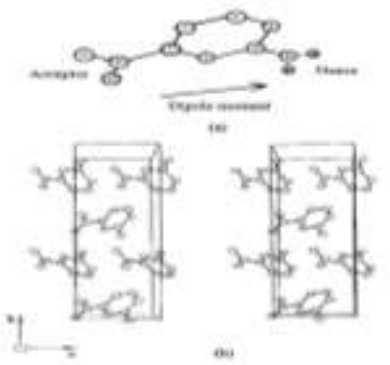

Figure 1 (a) Molecular structure of mNA

(b) Crystal packing diagram of mNA

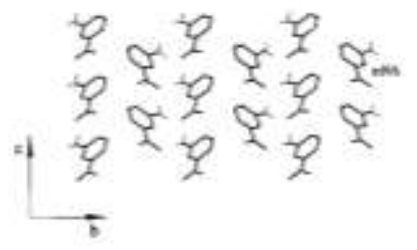

Figure 1(c) Projection of mNA crystal structure along the [100] direction. 


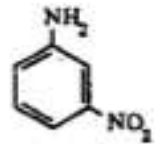

Figure 1(d) Chemical structure of mNA crystal

The arrangement of the molecules is consistent with the well known cleavage plane of mNA crystal because of the shortest intermolecular oxygen atom and the perpendicular distance between the planes of the mNA molecules stacked in b-direction is larger than any other intermolecular distances. This situation accounts for a very high nonlinear optical response in mNA molecule.

\section{CRYSTAL GROWTH}

The starting material was commercially available meta-nitroaniline (mNA) salt and its purity has been improved by recrystallizing in acetone for several times. The solvent evaporation technique was used to grow the single crystal of mNA. A recrystallized salt was dissolved in acetone, a saturated solution was prepared and the solution was filtered using a borozil filter paper. The filtered solution was taken in a beaker which was hermetically sealed to avoid the evaporation of the solvent. A good yellow colored single crystal of mNA was obtained in 20 days, is shown in Figure 2. The grown mNA crystals have been analyzed by different characterization techniques.

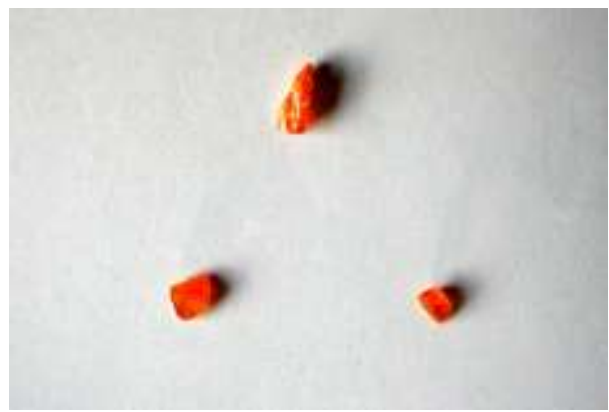

Figure 2. As grown mNA crystals from acetone

\section{CHARACTERIZATION STUDIES}

\subsection{Single crystal $X$-ray diffraction study}

Single crystal X-ray diffraction data for mNA were recorded by using ENRAF NONIUS CAD4 diffractometer. It is observed that the mNA single crystal belongs to orthorhombic crystal system with a space group $\mathrm{Pbc}_{21}$. The volume of the system $\mathrm{v}=647.7 \AA^{3}$ and the number of atom in the cell is $n=4$. The observed data are in very good agreement with previous determination and also these data for single crystal and powder XRD measurements are very well close with the reported values [16]

\subsection{FTIR analysis}

FTIR measurement was carried out in the range of 400-4000 cm-1 using the PERKIN ELMER RX1 Fourier Transform Infrared spectrometer prepared by mixing powdered mNA with $\mathrm{KBr}$ pellet technique is shown in Figure 3. For organic molecule, the FTIR region has been divided into fractional group and fingerprint region. The fractional group region extends from $4000 \mathrm{~cm}-1$ to $1300 \mathrm{~cm}-1$, whereas fingerprint region are those lying between 1340 to $900 \mathrm{~cm}-1$.The spectrum shows the symmetric and asymmetric stretching modes of free $\mathrm{NH}_{2}$ group, which are observed at 3229 and $3429.66 \mathrm{~cm}-1$. The peaks at 2280.91 cm-1 may be assigned to overtone of bonds due to 1263.05 and $1086.77 \mathrm{~cm}-1$. The vibration between 927.98 and $666.97 \mathrm{~cm}-1$ are usually associated with the presence of the benzene rings in the mNA molecule. The peaks between 734.61 and $867.9 \mathrm{~cm}-1$ show the meta position of the substituted molecules in the benzene ring of mNA [17]. The peaks that appear at 1349.04 $\mathrm{cm}-1$ are due to the vibrations of $\mathrm{NO}_{2}$ stretching modes which are not much broad, their interaction with the neighbouring molecules are presumed to be weak bonds. Nevertheless, the dipole-dipole interaction between the dominating forces in the packing of molecules in the crystal lattice.

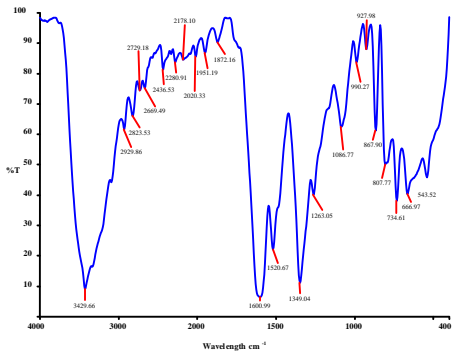

Fig.3. FTIR spectra for mNA

\subsection{UV-Visible transmittance study}

The UV-Vis-NIR transmission spectrum was recorded in the range of 300-1100 $\mathrm{nm}$ and is shown in the Figure 4. The instrument used was LAMBDA-35 UV-Vis spectrophotometer. From the spectrum, it is seen that the crystal has a lower cut-off wavelength of $410 \mathrm{~nm}$. The spectrum further indicates that the crystal has wide optical window from $410 \mathrm{~nm}$ to $1100 \mathrm{~nm}$. The crystal is transparent in the yellowish red and near infrared spectral regions. Optical transmittance of $70 \%$ was observed for $2 \mathrm{~mm}$ plates of mNA crystals. The crystal has good optical transmission in the visible region. The transparency in the visible region for this crystal suggests its suitability for second harmonic $g_{\imath}$

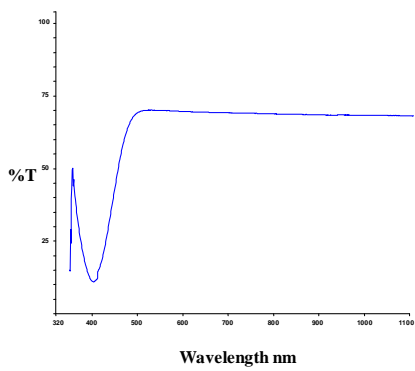

Figure 4. Optical transmittance spectra of mNA crystal

\subsection{Vickers hardness test}

Vickers microhardness indentation test is used to characterize the hardness of the material. Measurement of hardness is a nondestructive testing method to determine the mechanical behavior of the materials. It is a resistance against plastic deformation. By definition the indentation hardness is the ratio of the applied load to the surface area generated due to indentation. The hardness number can be evaluated by the knowledge of the load 
applied and the cross-sectional area of the depth of the impression. Smooth surfaces of as-grown mNA crystal were chosen for the investigation. The Vicker's hardness value is calculated from the formula $\mathrm{Hv}=1.8544 *\left(\mathrm{P} / \mathrm{d}^{2}\right) \mathrm{kg} / \mathrm{mm}^{2 \cdot}$ Where $\mathrm{P}$ is the applied load in $\mathrm{kg}$ and $\mathrm{d}$ is the average diagonal length in millimeters of the indented impressions. In the present study, hardness was measured using LeitzWetzler hardness tester. The loads applied are 5, 10, 25 and 50 grams and were applied at a time of 10 seconds. For each 25 and 50 gm, radial cracks were obtained for some indentation. This might be due to the plastic deformation or unique cracks induced in the mNA sample. Plot of load (P) against Vicker's hardness (Hv) is shown in the Figure 5. It was observed that the micro hardness number decreases with the increase of load in the given planes. It is noted that the hardness decreases as the load increases, which is in agreement with the normal indentation size effect (ISE) observed for other NLO crystals.

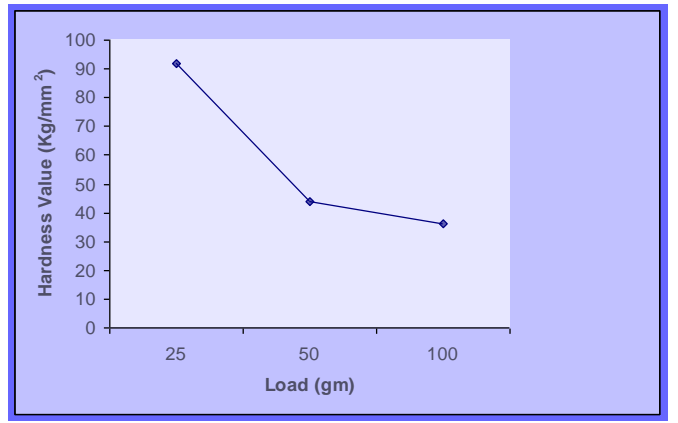

Figure 5. Load Vs. Hardness of mNA crystal

\subsection{SHG property test}

The SHG efficiency of the grown crystals was measured by Kurtz and Perry powder method [14]. The output from Nd: YAG laser $(\lambda=1064$ $\mathrm{nm}$ ) was used as source and it was illuminated to the powder form of mNA which was packed in a capillary tube of diameter $0.154 \mathrm{~mm}$. Pulse energy was $6.2 \mathrm{~mJ} / \mathrm{sec}$ and pulse width about $10 \mathrm{~ns}$ were used. The second harmonic signal $(532 \mathrm{~nm})$ of $91.66 \mathrm{~mW}$ and $1118.25 \mathrm{~mW}$ respectively were obtained for KDP and mNA samples. Microcrystalline materials of KDP are used for comparison with mNA for the SHG experiments. The output could be seen as a bright green flash emission from the sample. The green emission confirmed the second harmonic generation in the grown mNA crystal. Thus the SHG efficiency of mNA is higher than KDP.

\subsection{Etching studies}

Crystal defects may affect the properties of light absorption, scattering, refractive index, chemical homogeneity, electron mobility, mechanical strength and thermal stress. The defects were revealed using chemical etching. Chemical etching is able to develop some or all the features such as etch spirals, terraced, flat-bottomed pits, etch hillocks, etc., on a crystal surface. The etch pits are the result of chemical attack at the strain field surrounding the dislocation line. The shape and orientation of the pits can be related to crystal symmetry. In the present study, acetone is used as an etchant.The surface of as-grown crystal is shown in Figure 6 (a) for the etching on $\{100\}$ face produces a small circular pattern and striations shown in figure 6(b) for $5 \mathrm{sec}$. When the crystal was etched for $10 \mathrm{sec}$, the kinks and small-layered growth was observed as shown in figure 6(c). Also kinks and circular patterns were observed for $15 \mathrm{sec}$ etching time, as shown in figure 6(d). This may be due to change in diffusion field around a dislocation site with increase of etching time.

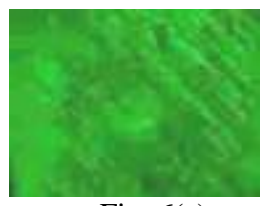

Fig. 6(a)

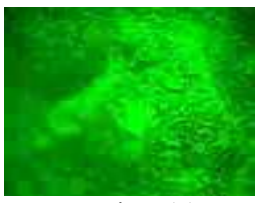

Fig.6(c)

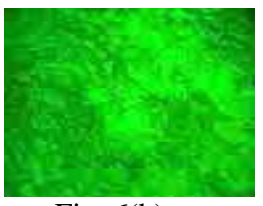

Fig. 6(b)

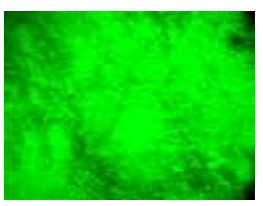

Fig. 6(d)
Etch patterns on Figure. 6 (a) (b) (c) (d)

\section{CONCLUSION}

The single crystals of mNA were successfully grown by slow evaporation technique using acetone as solvent. The lattice parameters have been determined from the single crystal X-ray diffraction pattern. The functional groups of the compound have been determined from the Fourier Transform infrared spectra. Optical tramittance study showed that the mNA crystal has good Optical Transmission in the entire visible region. The lower cut of wavelength falls at $410 \mathrm{~nm}$, which indicates mNA is a potential for SHG application. The second harmonic generation of the grown crystals was confirmed by Kurtz powder test using $\mathrm{Nd}$ : YAG laser as a source. The mechanical property was evaluated by Vickers micro hardness method. The straight striations and kinks were observed on the crystal surface during etching.

\section{REFERENCES}

[1] Ayer, S., Faktor, M.M, Marr, D and Stevenson, J.L. Mater. J.Sci. 7 (1972) 31-33.

[2] Bergman J.G., and Crane, G.R. J.Chem. Phys. 266 (1997) 3803-3805

[3] Chemla D.S., and Zyss, J. 'Nonlinear Optical Properties of Organic molecules and crystals', 1-2, Academic Press, Orlando, New York (1987).

[4] Chemla, D.S., Zyss, J. (Eds.), Nonlinear Optical Properties of Organic Molecules and Crystals, Academic Press, New York, 1987.

[5] Dmitriev, V.G., Gurzadyan, G.G. and Nikogosyan, D.N., in: Nonlinear Optical Crystals (Springer, Berlin, 1991) ch. 4.

[6] Gong-Fan Huang, Lin, J.T., Genbo Su, Rihong Jiang, Shengwu Xie, Opt. Commun., 89(1992) 205-211.

[7] Huang Bingrong, Su Genbo, He Youping, J. Crystal Growth, 102 (1990) 762-764.

[8] Ikeda, H., Sakai, T., Kawasaki, K. Chem. Phys. Lett. 179 (1991) 551.

[9] Kalymnios, D. J. Phys. D. Appl. Phys, 5(1972) 667669. 
[10] Katz, H.E., Singer, K.D., Sohn, J.E., Dirk, C.W., King, L.A., Gorden, H.M., J. Am. Chem.Soc. 109 (1987) 6561.

[11] Marder, S.R., Kippelen, B., Jen, A.K.-Y., Peyghambarian, N. Nature 388 (1997)845

[12] Nalva, H.S., Miyata, S. Nonlinear. Optics of Organic Molecules and Polymers, CRC Press, Boca Raton, 1997

[13] Prasad, P.N., Williams. D.J. introduction to Nonlinear Optical Effects in Organic Molecules and Polymers, Wiley, New York, 1991.

[14] Sharada and Mohan Rao, G.P., J. Crystal Growth, 210 (2000)824-827.
[15] Sreeramana Aithal, P., Mohan Rao. P., J3 Cryst. Growth 153 (1995) 60.

[16] Stevenson, J.L., and Skapsi. A.C., J. Phys. C: Solid State Phys., 5 (1972) 233.

[17] Szostak, M.M., Raman. J., Spec., 12 (1982) 228.

[18] Williams, V.J., in: Nonlinear Optical Properties of Organic and Polymeric Materials, m. Soc. Symp. Series 233 (American Chemical Society, Washington, DC, 1993) p.57. 